\title{
A Modified Algorithm for Generalized Discriminant Analysis
}

\author{
Wenming Zheng, Li Zhao, Cairong Zou \\ \{wenming_zheng, zhaoli, cairong\}@seu.edu.cn \\ The Engineering Research Center of Information Processing and Application, \\ Southeast University, Nanjing, Jiangsu 210096, P.R.China
}

Generalized discriminant analysis (GDA) is an extension of the classical linear discriminant analysis (LDA) from linear domain to a nonlinear domain via the kernel trick. However, in the previous algorithm of GDA, the solutions may suffer from the degenerate eigenvalue problem (i.e., several eigenvectors with the same eigenvalue), which makes them not optimal in terms of the discriminant ability. In this article, we propose a modified algorithm for GDA (MGDA) to solve this problem. The MGDA method aims to remove the degeneracy of GDA and find the optimal discriminant solutions, which maximize the between-class scatter in the subspace spanned by the degenerate eigenvectors of GDA. Theoretical analysis and experimental results on the ORL face database show that the MGDA method achieves better performance than the GDA method. 


\section{Introduction}

Linear discriminant analysis (LDA) (Duda \& Hart, 1973) is a well-known feature extraction method in pattern recognition. It finds the set of the optimal vectors that map the high-dimensional samples onto a low-dimensional feature space, where the ratio of the between-class scatter to the within-class scatter of the projected samples is extreme and the projected samples are well separated. Although the LDA method works for linear problem, it fails as for nonlinear case. Baudat and Anouar (2000) extended the LDA method from linear to a nonlinear domain using the kernel trick (Vapnik, 1995; Schölkopf, Smola, \& Müller, 1998) and then presented the Generalized Discriminant Analysis (GDA) method. The GDA aims to find the optimal discriminant nonlinear features for the training samples when they are not linearly separable. This is implemented by mapping the input space into a high-dimension (or even infinite-dimension) feature space via a nonlinear kernel function and performing the feature extractions using LDA in this feature space, thus producing the nonlinear features in the input space.

However, in using the Baudat-Anouar algorithm (Baudat \& Anouar, 2000) one may face a degenerate eigenvalue problem. This occurs especially in the case of the so-called small sample size (SSS) problem (Chen, Liao, Ko, Lin, \& Yu, 2000), where the most discriminant eigenvectors of GDA correspond to the same eigenvalue (i.e., degeneracy of eigenvectorsSchiff; Schiff, 1968).

In this paper, we modify the Baudat-Anouar algorithm and propose a robust and efficient algorithm to overcome the degenerate eigenvalue problem. The proposed 
algorithm aims to find the discriminant eigenvectors that maximize the between-class scatter matrix in the subspace spanned by the degenerate eigenvectors of GDA and thus remove the degeneracy of the solutions.

In the next section, we introduce a theorem used for this purpose. Then we review the GDA method. In section 3, we propose the MGDA method and develop the formulation. Section 4 is devoted to the experiments on the ORL face database. The discussion and conclusion are given in the last section.

\section{Related Work}

\section{A. Related Theorems}

Suppose that $S_{B}, S_{W}$ and $S_{T}$ are the between-class scatter matrix, the within-class scatter matrix and the total-class scatter matrix of the training samples, respectively. Let $I$ be the identity matrix.

Theorem 1 (Duchene, \& Leclercq, 1988) Let $\varphi_{1}, \cdots, \varphi_{r}$ be the first $r$ discriminant eigenvectors of Foley-Sammon Optimal Set of Discriminant Vectors (FSODV; Foley, \& Sammon, 1975), Then the $(r+1)$ th discriminant direction $\varphi_{r+1}$ of FSODV is the eigenvector corresponding to maximum eigenvalue of the eigenquation $P S_{B} \varphi=\lambda S_{W} \varphi$, where

$$
P=I-D^{T}\left(D S_{W}^{-1} D^{T}\right)^{-1} D S_{W}^{-1}, \quad D=\left[\begin{array}{llll}
\varphi_{1} & \varphi_{2} & \cdots & \varphi_{r}
\end{array}\right]^{T}
$$

Theorem 2 (Jin, Yang, Hu, \& Lou, 2001) Suppose that $\varphi_{1}, \cdots, \varphi_{r}$ are the first $r$ discriminant eigenvectors of the statistically uncorrelated optimal discriminant vectors 
(UODV; Jin, Yang, Hu, \& Lou, 2001). Then the $(r+1)$ th discriminant direction $\varphi_{r+1}$ of UODV is the eigenvector corresponding to maximum eigenvalue of the eigenequation $P S_{B} \varphi=\lambda S_{W} \varphi$, where

$$
P=I-S_{T} D^{T}\left(D S_{T} S_{W}^{-1} S_{T} D^{T}\right)^{-1} D S_{T} S_{W}^{-1}, \quad D=\left[\begin{array}{llll}
\varphi_{1} & \varphi_{2} & \cdots & \varphi_{r}
\end{array}\right]^{T}
$$

In fact, theorem 1 and theorem 2 can be extended to be a more general form. We give it as the theorem 3. ( The proof is given in appendix.)

Theorem 3 Suppose that $B$ and $R$ are positive semi-definite matrices, $V$ is a positive matrix. The discriminant criteria is defined as:

$$
F(\varphi)=\frac{\varphi^{T} B \varphi}{\varphi^{T} V \varphi}
$$

Let $\varphi_{1}$ be the discriminant eigenvector that maximizes $F(\varphi)$. Suppose that $\varphi_{i}$ $(i=1,2, \cdots, r \geq 1)$ are obtained. Let $\varphi_{r+1}$ be the $(r+1)$ th discriminant eigenvector that maximizes $F(\varphi)$ under the following constraints:

$$
\varphi_{r+1}^{T} R \varphi_{i}=0,(i=1,2, \cdots, r)
$$

Then, $\varphi_{r+1}$ is the eigenvector corresponding to the largest eigenvalue of the following eigenequation:

$$
P B \varphi=\lambda V \varphi
$$

where

$$
\begin{aligned}
& P=I-R D^{T}\left(D R V^{-1} R D^{T}\right)^{-1} D R V^{-1} \\
& D=\left[\begin{array}{llll}
\varphi_{1} & \varphi_{2} & \cdots & \varphi_{r}
\end{array}\right]^{T}
\end{aligned}
$$

\section{B. GDA Formulation in Kernel Space}

Suppose that $X$ is an $n$-dimensional sample set with $N$ elements. Let $X_{l}$ 
denote subset of $X$. Thus, $X=\bigcup_{l=1}^{c} X_{l}$, where $c$ is the number of the classes. The cardinality of the subsets $X_{l}$ is denoted by $N_{l}$. Thus, we have $\sum_{l=1}^{c} N_{l}=N$. Let $X$ be mapped into a Hilbert space $F$ through a nonlinear mapping function $\Phi$,

$$
\Phi: X \rightarrow F, x \rightarrow \Phi(x)
$$

The between-class scatter matrix $S_{B}^{\Phi}$, the within-class scatter matrix $S_{W}^{\Phi}$ and the total-scatter matrix $S_{T}^{\Phi}$ in $F$ are given as follows:

$$
\begin{aligned}
& S_{B}^{\Phi}=\sum_{i=1}^{c} N_{i}\left(u_{i}^{\Phi}-u^{\Phi}\right)\left(u_{i}^{\Phi}-u^{\Phi}\right)^{T} \\
& S_{W}^{\Phi}=\sum_{i=1}^{c} \sum_{j=1}^{N_{i}}\left(\Phi\left(x_{i}^{j}\right)-u_{i}^{\Phi}\right)\left(\Phi\left(x_{i}^{j}\right)-u_{i}^{\Phi}\right)^{T} \\
& S_{T}^{\Phi}=\sum_{i=1}^{c} \sum_{j=1}^{N_{i}}\left(\Phi\left(x_{i}^{j}\right)-u^{\Phi}\right)\left(\Phi\left(x_{i}^{j}\right)-u^{\Phi}\right)^{T}
\end{aligned}
$$

where $x^{T}$ represents the transpose of the vector $x, \Phi\left(x_{i}^{j}\right)$ is the $j$ th sample in the $i$ th class, $u_{i}^{\Phi}$ is the mean of the $i$ th class samples and $u^{\Phi}$ is the mean of all samples in $F$ :

$$
u_{i}^{\Phi}=\frac{1}{N_{i}} \sum_{j=1}^{N_{i}} \Phi\left(x_{i}^{j}\right), u^{\Phi}=\frac{1}{N} \sum_{i=1}^{c} \sum_{j=1}^{N_{i}} \Phi\left(x_{i}^{j}\right)
$$

GDAaims to find eigenvalues $\lambda$ and eigenvectors $\omega$, solutions of the equation

$$
S_{B}^{\Phi} \omega=\lambda S_{T}^{\Phi} \omega
$$

The largest eigenvalue of equation (2.11) gives the maximum of the following quotient of the inertia:

$$
\lambda=\frac{\omega^{T} S_{B}^{\Phi} \omega}{\omega^{T} S_{T}^{\Phi} \omega}
$$

Because the eigenvectors are linear combinations of $F$ elements, there exist coefficients $\alpha_{p q}\left(p=1, \cdots, c ; q=1, \cdots, N_{p}\right)$ such that 


$$
\omega=\sum_{p=1}^{c} \sum_{q=1}^{N_{p}} \alpha_{p q}\left(\Phi\left(x_{p}^{q}\right)-u^{\Phi}\right)
$$

Assume that a kernel function $k\left(x_{i}, x_{j}\right)$ can be expressed as the dot product form on the Hilbert space $F$ :

$$
k_{i j}=k\left(x_{i}, x_{j}\right)=<\Phi\left(x_{i}\right), \Phi\left(x_{j}\right)>=\left(\Phi\left(x_{i}\right)\right)^{T} \Phi\left(x_{j}\right)
$$

where $<\Phi\left(x_{i}\right), \Phi\left(x_{j}\right)>$ stands for the dot product of $\Phi\left(x_{i}\right)$ and $\Phi\left(x_{j}\right)$.

For a given classes $p$ and $q$, this kernel function can be expressed as:

$$
\left(k_{i j}\right)_{p q}=\left(\Phi\left(x_{p}^{i}\right)\right)^{T} \Phi\left(x_{q}^{j}\right)
$$

Let $W=\left(W_{l}\right)_{l=1, \cdots, c}$ be a $N \times N$ block diagonal matrix, where $W_{l}$ is a $N_{l} \times N_{l}$ matrix with all terms equal to $1 / N_{l}$. Let $M=\left(m_{i j}\right)_{i=1, \cdots, N ; j=1, \cdots, N}$ be a $N \times N$ matrix with all terms equal to $1 / N$ and let

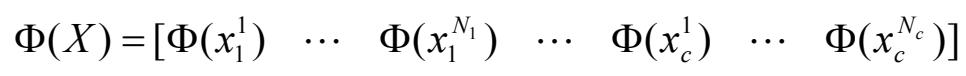

Then equation (2.13) can be expressed as

$$
\omega=\Phi(X)(I-M) \alpha
$$

where $\alpha=\left[\begin{array}{lllllll}\alpha_{11} & \cdots & \alpha_{1 N_{1}} & \cdots & \alpha_{c 1} & \cdots & \alpha_{c N_{c}}\end{array}\right]^{T}$.

Let $K$ be a $N \times N$ matrix defined on the class elements by $\left(K_{p q}\right)_{p=1, \cdots, c ; q=1, \cdots, c}$ :

$$
K=\left(K_{p q}\right)_{p=1, \cdots, c ; q=1, \cdots c}
$$

where $\left(K_{p q}\right)$ is a $N_{p} \times N_{q}$ matrix in the feature space $F$ :

$$
K_{p q}=\left(k_{i j}\right)_{p q}, \quad i=1, \cdots, N_{p}, \quad j=1, \cdots, N_{q}
$$

Using the notations above, the equations (2.11), (2.12) and (2.18) are equivalent to the following expressions, respectively:

$$
B \alpha=\lambda T \alpha
$$




$$
\begin{gathered}
\lambda=\frac{\alpha^{T} B \alpha}{\alpha^{T} T \alpha} \\
K=(\Phi(X))^{T} \Phi(X)
\end{gathered}
$$

where

$$
\begin{gathered}
B=(I-M)^{T} K(W-M)(W-M)^{T} K(I-M) \\
T=(I-M)^{T} K(I-M)(I-M)^{T} K(I-M)
\end{gathered}
$$

Baudat and Anouar (2000) give a method to resolve the eigenequation (2.20) by using the eigenvectors decomposition method (for more details of the algorithm, see Baudat \& Anouar, 2000). Although that method can resolve the eigenvectors and the corresponding eigenvalues of the eigenequation (2.20) very well, there is a weakness containing in it which still has not been overcome yet. This is the degenerate perturbation problem of the eigenvectors (i.e., several eigenvectors with the same eigenvalue). This problem often occurs in many cases such as the small sample size problem: Denote the null space of $S_{W}^{\Phi}$ by $S_{W}^{\Phi}(0)$. As for small sample size problem, suppose the solution $\omega \in S_{W}^{\Phi}(0)$, then we have $S_{W}^{\Phi} \omega=0$. Note that $S_{T}^{\Phi}=S_{W}^{\Phi}+S_{B}^{\Phi}$, thus we have

$$
\omega^{T} S_{T}^{\Phi} \omega=\omega^{T} S_{W}^{\Phi} \omega+\omega^{T} S_{B}^{\Phi} \omega=\omega^{T} S_{B}^{\Phi} \omega
$$

From equations (2.17) and (2.25), we have

$$
\alpha^{T} T \alpha=\alpha^{T} B \alpha \Rightarrow \frac{\alpha^{T} B \alpha}{\alpha^{T} T \alpha}=1
$$

From equation (2.26), we obtain that all the eigenvectors in the null space of the within-class scatter matrix share the same maximal eigenvalue $(=1)$. 


\section{Modified Algorithm for GDA}

The GDA method provides an efficient technique to calculate the discriminant eigenvectors in the feature space $F$. However, in many cases such as the small sample size problem, some of the eigenvectors solved by GDA may be degenerate.

Suppose that $\alpha_{k}^{(1)}, \cdots, \alpha_{k}^{(t)}(t>1)$ are the degenerate eigenvectors of the eigenequation (2.20) sharing the same eigenvalue $\lambda_{k}$, i.e.

$$
B \alpha_{k}^{(i)}=\lambda_{k} T \alpha_{k}^{(i)}, \quad i=1, \cdots, t
$$

From the equations (2.11) and (2.17), equation (3.1) is equivalent to the expressions:

$$
S_{B}^{\Phi} \omega_{k}^{(i)}=\lambda_{k} S_{T}^{\Phi} \omega_{k}^{(i)}, \quad i=1, \cdots, t
$$

where

$$
\omega_{k}^{(i)}=\Phi(X)(I-M) \alpha_{k}^{(i)}, \quad i=1, \cdots, t
$$

Thus, $\omega_{k}^{(i)}(i=1, \cdots, t)$ are the eigenvectors corresponding to the same eigenvalue $\lambda_{k}$ of the eigenequation (2.11). Let $\Omega$ denote the subspace spanned by the eigenvectors $\omega_{k}^{(i)}(i=1, \cdots, t)$. Then any vector in $\Omega$ is the eigenvector of the eigenequation (2.11) corresponding to the eigenvalue $\lambda_{k}$. Thus, the solutions of GDA may be unstable with respect to changes in training data (model variance) and probably are not optimal in terms of the discriminant ability. The MGDA method overcomes this problem by limiting the attention to the subspace $\Omega$ to find the eigenvectors with the best discriminant ability.

Suppose that $\widetilde{\omega}_{k}^{(i)} \in \Omega(i=1, \cdots, t)$ are the eigenvectors of the eigenequation (2.11) with best discriminant ability, where 


$$
\widetilde{\omega}_{k}^{(i)}=\sum_{j=1}^{t} \omega_{k}^{(j)} \gamma_{i j}(i=1, \cdots, t)
$$

Let $A=\left[\begin{array}{lll}\alpha_{k}^{(1)} & \cdots & \alpha_{k}^{(t)}\end{array}\right]$ and $\gamma_{i}=\left[\begin{array}{lll}\gamma_{i 1} & \cdots & \gamma_{i t}\end{array}\right]^{T}$. Then equation (3.4) can be rewritten as:

$$
\widetilde{\omega}_{k}^{(i)}=\Phi(X)(I-M) A \gamma_{i}, \quad i=1, \cdots, t
$$

According to the physical meaning of discriminant analysis (Fukunaga, 1990), the projections of the training samples projected by the solutions $\widetilde{\omega}_{k}^{(i)}(i=1, \cdots, t)$ should have maximal between-class scatter in order to get better discriminant ability. To do this, we should define new discriminant criteria to replace the previous discriminant criteria (equation 2.12). Such a criteria can be expressed as:

$$
J_{1}(\omega)=\frac{\omega^{T} S_{B}^{\Phi} \omega}{\omega^{T} \omega}, \quad \text { where } \omega \in \Omega
$$

By using the new criteria, these discriminant eigenvectors can be generated in the following forms: the first eigenvector $\widetilde{\omega}_{k}^{(1)}$ is the one that maximizes $J_{1}(\omega)$ in $\Omega$; Suppose that the first $r$ discriminant eigenvectors $\widetilde{\omega}_{k}^{(i)}=\Phi(X)(I-M) A \gamma_{i}$ $(i=1,2, \cdots, r<t)$ are obtained, then the $(r+1)$ th eigenvector $\widetilde{\omega}_{k}^{(r+1)}$ is the one that maximizes $J_{1}(\omega)$ in $\Omega$ under the following orthogonal constraints:

$$
\left(\widetilde{\omega}_{k}^{(r+1)}\right)^{T} \widetilde{\omega}_{k}^{(i)}=0, i=1,2, \cdots, r
$$

From the equation (3.5), we obtain that to find $\widetilde{\omega}_{k}^{(1)}$ is equivalent to find the coefficient $\gamma_{1}$ that maximizes $J_{2}(\gamma)$, where

$$
\begin{aligned}
& J_{2}(\gamma)=\frac{\gamma^{T} \widetilde{B} \gamma}{\gamma^{T} R \gamma} \\
& \widetilde{B}=A^{T} B A \\
& R=A^{T}(I-M)^{T} K(I-M) A
\end{aligned}
$$


From discriminant analysis (Duda, \& Hart, 1973; Fukunaga, 1990): $\gamma_{1}$ is the eigenvector of the following generalized eigenequation corresponding to the largest eigenvalue:

$$
\widetilde{B} \gamma=\lambda R \gamma
$$

By the same method, to find the $(r+1)$ th eigenvector $\widetilde{\omega}_{k}^{(r+1)}$ that maximizes $J_{1}(\omega)$ under the orthogonal constraints of equation (3.7) in $\Omega$ is equivalent to find the coefficient $\gamma_{r+1}$ that maximizes $J_{2}(\gamma)$ under the following constraints:

$$
\gamma_{r+1}^{T} R \gamma_{i}=0, i=1,2, \cdots, r
$$

From theorem 3, we obtain that $\gamma_{r+1}$ is the eigenvector corresponding to the largest eigenvalue of the following eigenequation:

$$
P \widetilde{B} \gamma=\lambda R \gamma
$$

where

$$
\begin{aligned}
& P=I-R D^{T}\left(D R D^{T}\right)^{-1} D \\
& D=\left[\begin{array}{llll}
\gamma_{1} & \gamma_{2} & \cdots & \gamma_{r}
\end{array}\right]^{T}
\end{aligned}
$$

The coefficient $\gamma_{i}(i=1, \cdots, t)$ is normalized by requiring that the corresponding eigenvector $\widetilde{\omega}_{k}^{(i)}(i=1, \cdots, t)$ is normalized in $F$, i.e.

$$
\left(\widetilde{\omega}_{k}^{(i)}\right)^{T} \widetilde{\omega}_{k}^{(i)}=1
$$

Using equations (2.22) and (3.5), we have

$$
\gamma_{i}^{T} A^{T}(I-M)^{T} K(I-M) A \gamma_{i}=1
$$

Thus the coefficient $\gamma_{i}$ is divided by $\sqrt{\gamma_{i}^{T} A^{T}(I-M)^{T} K(I-M) A \gamma_{i}}$ in order to get normalized eigenvector $\widetilde{\omega}_{k}^{(i)}$.

The MGDA procedure can be summarized in the following steps: 
1. Compute the discriminant vectors by using the Baudat-Anouar's algorithm. If no degeneracy occurs, then finish the algorithm and the solutions of MGDA equal to those of GDA; else go to step 2.

2. Select the discriminant vectors $\alpha_{k}^{(i)}(i=1, \cdots, t)$ corresponding to the same eigenvalue $\lambda_{k}$, where $t$ is the number of the degenerate vectors.

3. Compute the matrices $\widetilde{B}$ and $R$ (see equations 3.9 and 3.10).

4. Compute the eigenvector $\gamma_{1}$ using system 3.11 .

5. Compute the eigenvectors $\gamma_{i}(i=2, \cdots, t)$ using system 3.13.

6. Compute eigenvectors $\widetilde{\omega}_{k}^{(i)}$ using $\gamma_{i}(i=1, \cdots, t)$ (see equation 3.5) and normalize them (see equation 3.17).

\section{Experiments}

We test the MGDA method on the Olivetti Research Lab. (ORL) face database in Cambridge (Online Available: http://www.cam-orl.co.uk/facedatabase.html). The ORL database contains 40 distinct subjects, where each one contains 10 different images taken at different times, varying lighting slightly. All the images are taken against a dark homogeneous background and the persons are in upright, frontal position, with tolerance for some tilting and rotation. Figure 1 shows ten face images of one subject in the face database. The original face images are all sized $112 \times 92$ pixels with a 256-level gray scale. For each image, we use a two-level wavelet transform (Chien \& Wu, 2002) and get a low-pass image of $28 \times 23$ size pixels. 
Then we normalize the intensity values of the low-pass image with a linear function. After doing that, the dimension of the image vector is 644 . The mean and standard deviation of Kurtosis of the images are 2.08 and 0.39 , respectively. The polynomial and gaussian kernels used in the experiments are defined as (Baudat \& Anouar, 2000):

Polynomial kernel: $k(x, y)=(<x, y>)^{d}$, where $d$ is the polynomial degree;

Gaussian kernel: $k(x, y)=\exp \left(-\|x-y\|^{2} / \sigma\right)$, where the parameter $\sigma$ has to be chosen.
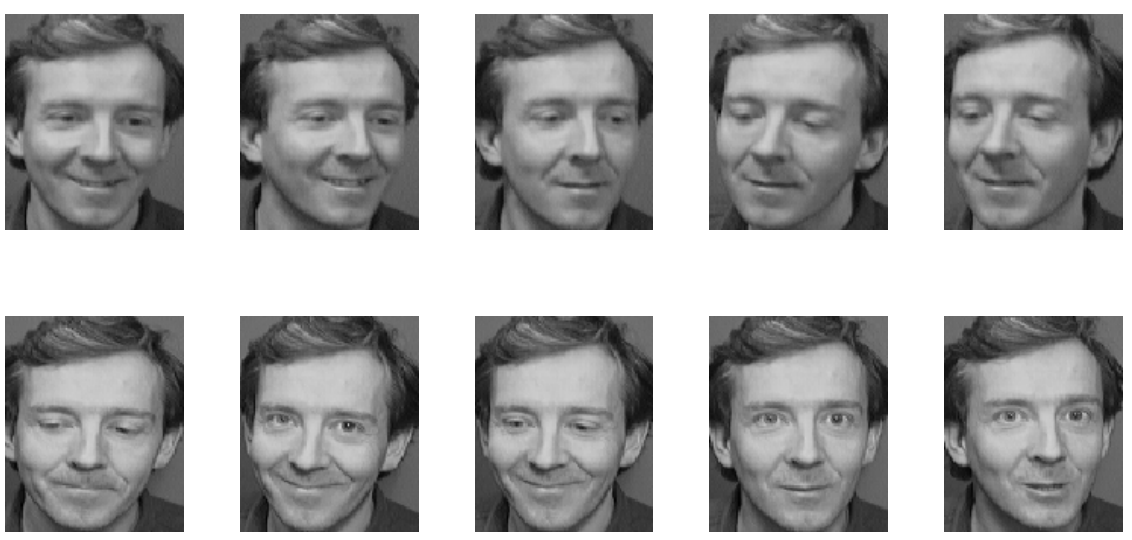

Figure 1: Ten face images for one subject in ORL face database

\subsection{Face Recognition}

Two examples for face recognition based on the nearest neighbor classifier are performed in this experiment. The first example is similar with that done by Yang (2002), which aims to compare the performance of GDA and MGDA with other methods in face recognition. We use the leave-one-out strategy to perform this experiment: To classify an face image, we remove it from the whole face image set, and the discriminant vectors are computed using the training set of the remainder 399 
images. The test image and the training images then are projected to a reduced space using the computed discriminant vectors of GDA and MGDA, respectively. Table 1 shows the experimental results. We can see from Table 1 that the MGDA method achieves the error rate as low as the Kernel Fisherface method $(=1.25 \%)$, which is the lowest error rate among the methods reported by Yang (2002). We also see that the MGDA method achieves better performance than the GDA method in this example.

Table 1 Performance of various systems

\begin{tabular}{|l|c|c|}
\hline \multicolumn{1}{|c|}{ Method } & Reduced Space & Error Rate (\%) \\
\hline Eigenface (Yang, 2002) & 40 & $2.50(10 / 400)$ \\
\hline Fisherface (Yang, 2002) & 39 & $1.50(6 / 400)$ \\
\hline SVM (Yang, 2002) & N/A & $3.00(12 / 400)$ \\
\hline Kernel Eigenface (Yang, 2002) & 40 & $2.00(8 / 400)$ \\
\hline Kernel Fisherface (Yang, 2002) & 39 & $1.25(5 / 400)$ \\
\hline GDA (Polynomial kernel with $d=2)$ & 39 & $2.25(9 / 400)$ \\
\hline MGDA (Polynomial kernel with $d=2)$ & 39 & $1.5(6 / 400)$ \\
\hline GDA (Gaussian kernel with $\sigma=10000)$ & 39 & $1.25(5 / 400)$ \\
\hline MGDA (gaussian kernel with $\sigma=10000)$ & $39 / 400)$ \\
\hline
\end{tabular}

The second example aims to further to compare the performance of GDA and MGDA in face recognition. Ten images per subject are randomly partitioned into five training images and five test images for a total of 200 training images and 200 test 
images. There is no overlap between the two sets. Two trials of tests are performed by swapping the training and the test sets. We treat each test as a Bernoulli random test and the average test error rate as the probability of the wrong classification over all the 400 tests. Table 2 shows the average test error rate and the standard deviation for each method, where MLDA is a particular case of GDA using the polynomial kernel with the degree $d=1$. As can be seen form Table 2, the MGDA achieves lower average test error rate and standard deviation than GDA over all the tests.

Table 2: Average test error rate for GDA and MGDA

\begin{tabular}{|c|c|c|c|c|c|c|}
\hline Method & LDA & MLDA & GDA $^{1)}$ & MGDA $^{1)}$ & GDA $^{2)}$ & MGDA $^{2)}$ \\
\hline $\begin{array}{c}\text { Average Test } \\
\text { Error Rate(\%) }\end{array}$ & 10.0 & 5.0 & 4.75 & 3.0 & 4.25 & 2.75 \\
\hline $\begin{array}{c}\text { Standard } \\
\text { Deviation }\end{array}$ & 6.0 & 4.3589 & 4.2541 & 3.4117 & 4.0345 & 3.2707 \\
\hline
\end{tabular}

Note: ${ }^{1)}$ polynomial kernel with $d=2$ is used; ${ }^{2)}$ gaussian kernel with $\sigma=10000$ is used.

\subsection{Stability Test}

This experiment aims to compare the performance of stability between GDA and MGDA. We select five images randomly from per subject as training samples and use the other five images as test images, thus obtain 200 training images and 200 test images. The gaussian kernel with $\sigma=10000$ and the nearest neighbor classifier are used in this experiment.

Suppose that $\omega_{i}$ and $\widetilde{\omega}_{i}(i=1, \cdots, 39)$ are the 39 discriminant eigenvectors computed by GDA and MGDA, respectively. Let $Z_{G D A}=\left[\begin{array}{lll}\omega_{1} & \cdots & \omega_{39}\end{array}\right]$ and let 
a)

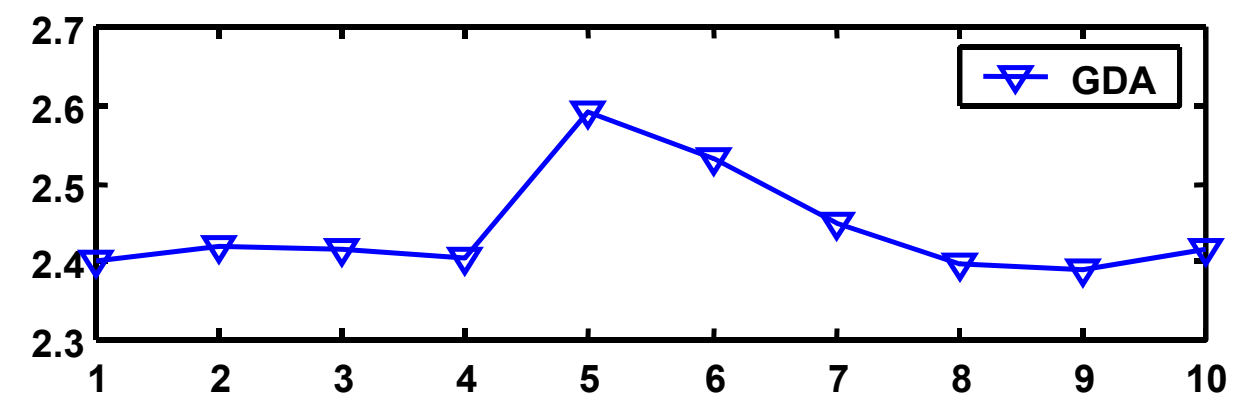

b)

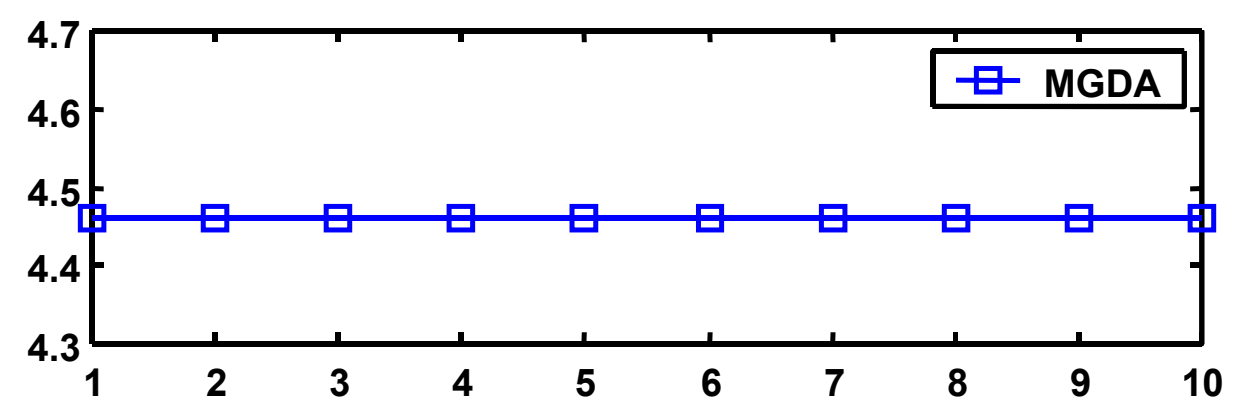

c)

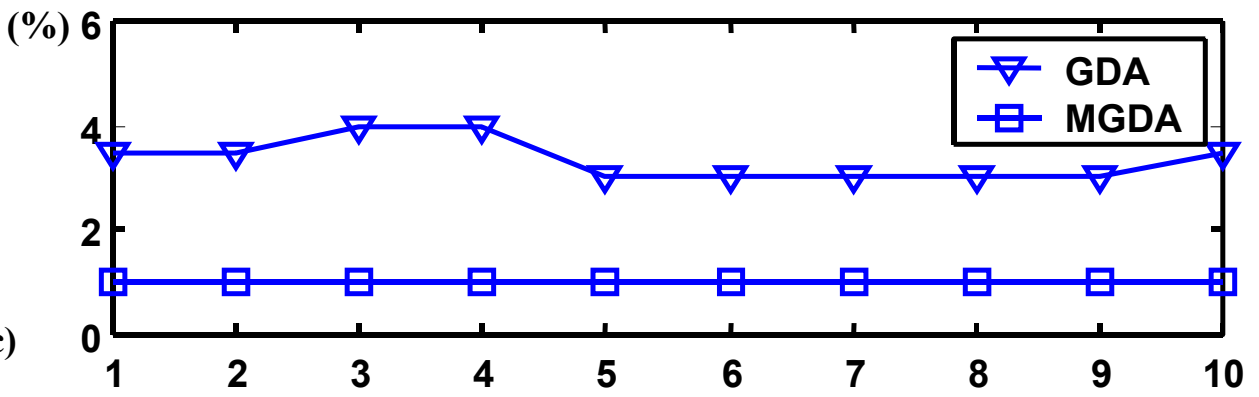

Figure 2: The comparison of the stability between GDA and MGDA. (a) The value of $\Psi\left(Z_{G D A}\right)$ in each trial; (b) The value of $\Psi\left(Z_{M G D A}\right)$ in each trial; (c) The test error rate of GDA and MGDA in each trial.

$Z_{M G D A}=\left[\begin{array}{lll}\widetilde{\omega}_{1} & \cdots & \widetilde{\omega}_{39}\end{array}\right]$. We re-order the training images in each subject and then re-compute the projected matrices $Z_{G D A}$ and $Z_{M G D A}$. Ten trials are repeated in this experiment. In each trial, we write down the value of $\Psi\left(Z_{G D A}\right)$ and $\Psi\left(Z_{M G D A}\right)$, respectively, where $\Psi(Z)=\operatorname{tr}\left(Z^{T} S_{B}^{\Phi} Z\right)$. We also use the 200 test images to perform face recognition using the projected matrices $Z_{G D A}$ and $Z_{M G D A}$, respectively. Figure 
2 shows the experimental results of the ten trials. From Figure 2, we can see that the values of $\Psi\left(Z_{M G D A}\right)$ and the corresponding test error rate are stable over all the ten trials for MGDA. However, it is not the case for GDA. As for GDA, the values of $\Psi\left(Z_{G D A}\right)$ have slight change over all the trials and the test error rates also have small change in some trials.

\section{Disscussion and Conclusion}

GDA method is the generalization for LDA method as nonlinear discrimination analysis via the kernel trick. Baudat and Anouar (2000) provide an algebra formulation and the eigenvalue resolution, which can give an exact solution for GDA even if some points, such as the choice of kernel function, require further investigation. However, the further study for GDA shows us that the resolution method for GDA Baudat and Anouar (2000) developed may suffer from instability and inaccuracy due to the degenerate perturbation of the solutions, especially to the small sample size problem where the most discriminant eigenvectors in the null space of the within-class scatter matrix share the same maximal eigenvalue $(=1)$. In this paper, we have developed a modified algorithm for GDA (MGDA), to overcome this problem. The performance of MGDA is just the same as GDA when no degeneracy occurs. If degeneracy occurs, our theoretical analysis and the experiments based on the ORL face database show that the MGDA method still keeps good performance and is superior to the GDA method. 
Besides, in the first example in section 4.1, we see that the MGDA method gets exactly the same performance as the Kernel Fisherface method. In fact, we can see that the Kernel Fisherface algorithm developed by Yang (2002) and the GDA algorithm by Baudat and Anouar (2000) are two different algorithms based on the same criteria (equation 2.12) which solves the discriminant analysis problems in feature space. However, both methods did not overcome the possible degenerate problem of the solutions. Thus, the best performance of MGDA could be exactly equal to that of Kernel Fisherfaces.

\section{Appendix A}

\section{Proof of theorem 3:}

Proof. Let $\widetilde{\varphi}_{r+1}$ be the direction of $\varphi_{r+1}$, and $\widetilde{\varphi}_{r+1}$ satisfies the following constraint

$$
\widetilde{\varphi}_{r+1}^{T} V \widetilde{\varphi}_{r+1}=1
$$

Thus, from equation (2.2), we have

$$
\widetilde{\varphi}_{r+1}^{T} R \varphi_{i}=0, \quad i=1, \cdots, r
$$

In order to compute $\widetilde{\varphi}_{r+1}$, we use the method of Lagrange multipliers to transform the criteria (2.1) including all the constrains

$$
L\left(\widetilde{\varphi}_{r+1}\right)=\widetilde{\varphi}_{r+1}^{T} B \widetilde{\varphi}_{r+1}-\lambda\left[\widetilde{\varphi}_{r+1}^{T} V \widetilde{\varphi}_{r+1}-1\right]-\sum_{i=1}^{r} \mu_{i} \widetilde{\varphi}_{r+1}^{T} R \varphi_{i}
$$

where $\lambda$ and $\mu_{i}, i=1,2, \cdots, r$ are Lagrange multipliers.

The optimization is performed by setting the partial derivative of $L\left(\widetilde{\varphi}_{r+1}\right)$ with respect to $\tilde{\varphi}_{r+1}$ equal to zero: 


$$
2 B \widetilde{\varphi}_{r+1}-2 \lambda V \widetilde{\varphi}_{r+1}-\sum_{i=1}^{r} \mu_{i} R \varphi_{i}=\mathbf{0}
$$

Multiplying the left-hand side of equation (A.4) by $\widetilde{\varphi}_{r+1}^{T}$, and from equation (A.2), we obtain that:

$$
\lambda=\frac{\widetilde{\varphi}_{r+1}^{T} B \widetilde{\varphi}_{r+1}}{\widetilde{\varphi}_{r+1}^{T} V \widetilde{\varphi}_{r+1}}=F\left(\widetilde{\varphi}_{r+1}\right)
$$

Thus, $\lambda$ represents the expression $F\left(\widetilde{\varphi}_{r+1}\right)$ to be maximized.

Multiplying the left-hand side of equation (A.4) by $\varphi_{j}^{T} R V^{-1}, j=1,2, \cdots, r$, we obtain a set of $r$ expression:

$$
2 \varphi_{j}^{T} R V^{-1} B \widetilde{\varphi}_{r+1}-\sum_{i=1}^{r} \mu_{i} \varphi_{j}^{T} R V^{-1} R \varphi_{i}=0, j=1,2, \cdots, r
$$

or in another form:

$$
\begin{aligned}
& 2 \varphi_{1}^{T} R V^{-1} B \widetilde{\varphi}_{r+1}-\sum_{i=1}^{r} \mu_{i} \varphi_{1}^{T} R V^{-1} R \varphi_{i}=0 . \\
& 2 \varphi_{2}^{T} R V^{-1} B \widetilde{\varphi}_{r+1}-\sum_{i=1}^{r} \mu_{i} \varphi_{2}^{T} R V^{-1} R \varphi_{i}=0 . \\
& \ldots \\
& 2 \varphi_{r}^{T} R V^{-1} B \widetilde{\varphi}_{r+1}-\sum_{i=1}^{r} \mu_{i} \varphi_{r}^{T} R V^{-1} R \varphi_{i}=0 .
\end{aligned}
$$

i.e.

$$
2\left[\begin{array}{c}
\varphi_{1}^{T} \\
\varphi_{2}^{T} \\
\vdots \\
\varphi_{r}^{T}
\end{array}\right] R V^{-1} B \widetilde{\varphi}_{r+1}-\left[\begin{array}{c}
\varphi_{1}^{T} \\
\varphi_{2}^{T} \\
\vdots \\
\varphi_{r}^{T}
\end{array}\right] R V^{-1} R\left[\begin{array}{c}
\varphi_{1}^{T} \\
\varphi_{2}^{T} \\
\vdots \\
\varphi_{r}^{T}
\end{array}\right]^{T}\left[\begin{array}{c}
\mu_{1} \\
\mu_{2} \\
\vdots \\
\mu_{r}
\end{array}\right]=0
$$

Let

$$
\boldsymbol{\mu}=\left[\begin{array}{llll}
\mu_{1} & \mu_{2} & \cdots & \mu_{r}
\end{array}\right]^{T}
$$

Considering the matrix notation (2.5), the previous set of $r$ equations (A.8) can be represented in a single matrix relation: 


$$
D R V^{-1} R D^{T} \boldsymbol{\mu}=2 D R V^{-1} B \widetilde{\varphi}_{r+1}
$$

Thus, we obtain

$$
\boldsymbol{\mu}=2\left(D R V^{-1} R D^{T}\right)^{-1} D R V^{-1} B \widetilde{\varphi}_{r+1}
$$

It is obvious that the following equation holds:

$$
\sum_{i=1}^{r} \mu_{i} R \varphi_{i}=R D^{T} \boldsymbol{\mu}
$$

Thus, equation (A.4) can be written as the following form:

$$
2 B \widetilde{\varphi}_{r+1}-2 \lambda V \widetilde{\varphi}_{r+1}-R D^{T} \boldsymbol{\mu}=0
$$

Substituting (A.11) into (A.13), we have

$$
2 B \widetilde{\varphi}_{r+1}-2 \lambda V \widetilde{\varphi}_{r+1}-R D^{T}\left[2\left(D R V^{-1} R D^{T}\right)^{-1} D R V^{-1} B \widetilde{\varphi}_{r+1}\right]=0
$$

or in another form

$$
B \widetilde{\varphi}_{r+1}-R D^{T}\left(D R V^{-1} R D^{T}\right)^{-1} D R V^{-1} B \widetilde{\varphi}_{r+1}=\lambda V \widetilde{\varphi}_{r+1}
$$

i.e.

$$
\left[I-R D^{T}\left(D R V^{-1} R D^{T}\right)^{-1} D R V^{-1}\right] B \widetilde{\varphi}_{r+1}=\lambda V \widetilde{\varphi}_{r+1}
$$

Considering the matrix notation (2.4), the equation (A.16) can be expressed as

$$
P B \widetilde{\varphi}_{r+1}=\lambda V \widetilde{\varphi}_{r+1}
$$

Because that $\widetilde{\varphi}_{r+1}$ is the direction of $\varphi_{r+1}$, we therefore obtain that

$$
P B \varphi_{r+1}=\lambda V \varphi_{r+1}
$$

Besides, it is noted that $\varphi_{r+1}$ is unitary vector, thus we have

$$
\varphi_{r+1}^{T} \varphi_{r+1}=1
$$

From the equations (A.18) and (A.19), we know that $\varphi_{r+1}$ is the eigenvector corresponding to the largest eigenvalue $\lambda$ of the eigenequation (A.18). 


\section{References}

Baudat G., \& Anouar F. (2000), “Generalized discriminant analysis using a kernel approach,” Neural Computation, vol.12, pp.2385-2404.

Chen, L.F., Liao, H.Y.M., Ko, M.T., Lin, J.C., \& Yu, G.J. (2000), “A new LDA-based face recognition system which can solve the small sample size problem," Pattern Recognition, 33, pp.1713-1726.

Chien, J.T. \& Wu, C.C. (2002), “Discriminant Waveletfaces and Nearest Feature Classifiers for Face Recognition", IEEE Transactions on Pattern Analysis and Machine Intelligence, Vol. 24, No. 12, pp.1644-1649.

Duchene, J., \& Leclercq, A. (1988), “An Optimal Transformation for Discriminant and Principal Component Analysis", IEEE Transactions on Pattern Analysis and Machine Intelligence, Vol.10, No.6, pp. 978-983.

Duda, R.O., \& Hart, P.E. (1973), "Pattern Classification and Scene Analysis”, New York: John Wiley \& Sons, Inc.

Foley, D.H., \& Sammon, J.W., JR. (1975), “An Optimal Set of Discriminant Vectors,” IEEE Trans. on Computer, vol. C-24, No. 3, pp.281-289.

Fukunaga, K. (1990), “Introduction to Statistical Pattern Recognition”, Academic Press, Inc.

Jin, Z., Yang, J.Y., Hu, Z.S., \& Lou, Z., (2001), "Face recognition based on the uncorrelated discriminant transformation," Pattern Recognition, 34, pp. 1405-1416.

Schiff L. (1968), “Quantum Mechanics”, $3^{\text {rd }}$ ed., McGraw-Hill, New York.

Schölkopf, B., Smola, A., \& Müller, K.R. (1998), "Nonlinear component analysis as a 
kernel eigenvalue problem,” Neural Computation, vol.10, pp.1299-1319.

Vapnik, V. (1995), “The Nature of Statistical Learning Theory: Springer, 1995.

Yang, M.H. (2002), “Kernel Eigenfaces vs. Kernel Fisherfaces: Face Recognition

Using Kernel Methods", Proceedings of the Fifth International conference on Automatic Face and Gesture Recognition (FG 2002), Washington D. C., May, IEEE Computer Society, pp.215-220. 